Supporting Information

\title{
Fe-Supported SBA-16 Type Cagelike Mesoporous Silica with Enhanced Catalytic Activity for Direct Hydroxylation of Benzene to Phenol
}

Milad Jourshabani ${ }^{1,2}$, Alireza Badiei ${ }^{1,3^{*}}$, Zahra Shariatinia $^{2}$, Negar Lashgari ${ }^{1}$, Ghodsi Mohammadi Ziarani ${ }^{4}$

${ }^{1}$ School of Chemistry, College of Science, University of Tehran, P.O. Box 14155-6455, Tehran, Iran

${ }^{2}$ Department of Chemistry, Amirkabir University of Technology, P.O. Box 159163-4311, Tehran, Iran

${ }^{3}$ Nanobiomedicine Center of Excellence, Nanoscience and Nanotechnology Research Center, University of Tehran, Tehran, Iran

${ }^{4}$ Department of Chemistry, Alzahra University, P.O. Box 1993893973, Tehran, Iran 


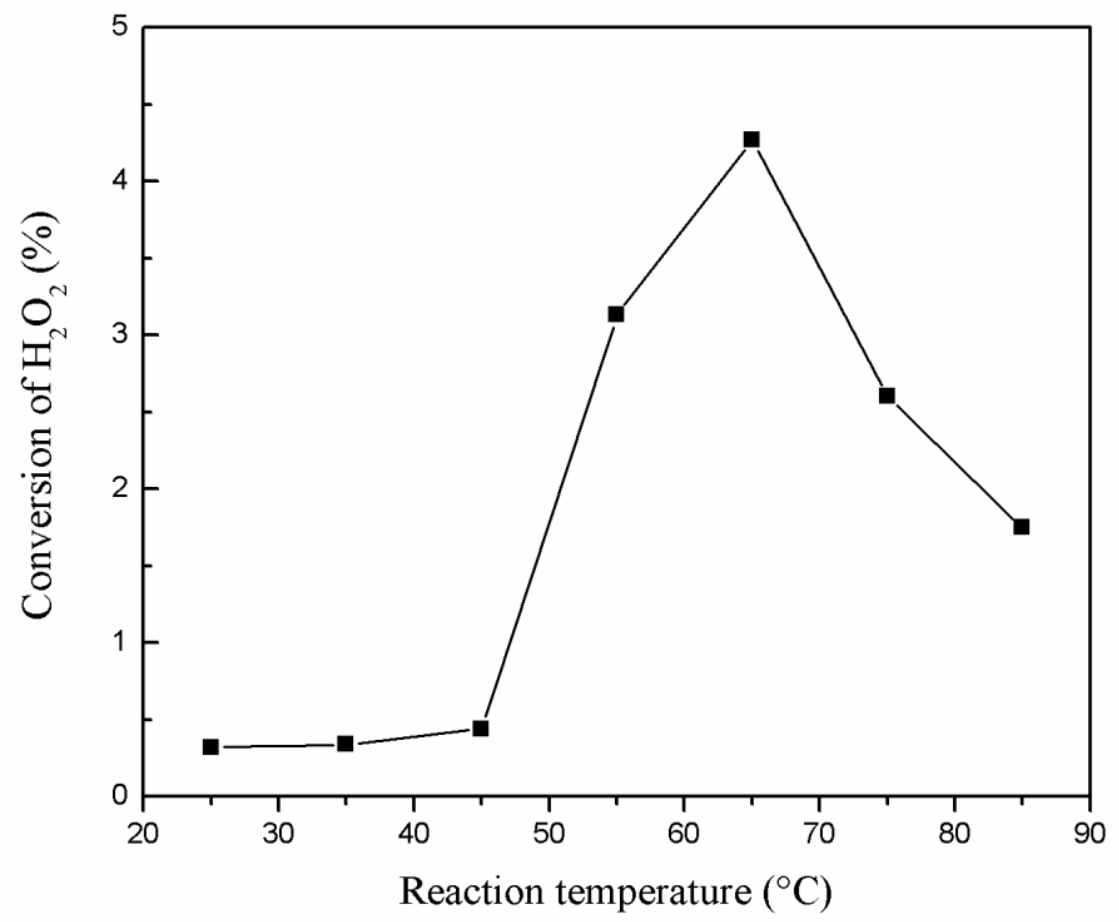

Figure S1. Effect of the reaction temperature on the conversion of $\mathrm{H}_{2} \mathrm{O}_{2}$. Reaction conditions: benzene (1.0 mL, $11.26 \mathrm{mmol}), 30 \%$ aq $\mathrm{H}_{2} \mathrm{O}_{2}(2 \mathrm{~mL}, 20 \mathrm{mmol}), \mathrm{Fe} / \mathrm{SBA}-16(0.1$ $\mathrm{g}$ ), acetonitrile $(6 \mathrm{~mL}, 114.8 \mathrm{mmol}), \mathrm{t}=8 \mathrm{~h}$. Conversion of $\mathrm{H}_{2} \mathrm{O}_{2}$ calculated as (mole phenol + $2 \times$ mole hydroquinone $+2 \times$ mole 1, 4-benzoquinone) /initial mole of $\mathrm{H}_{2} \mathrm{O}_{2}$.

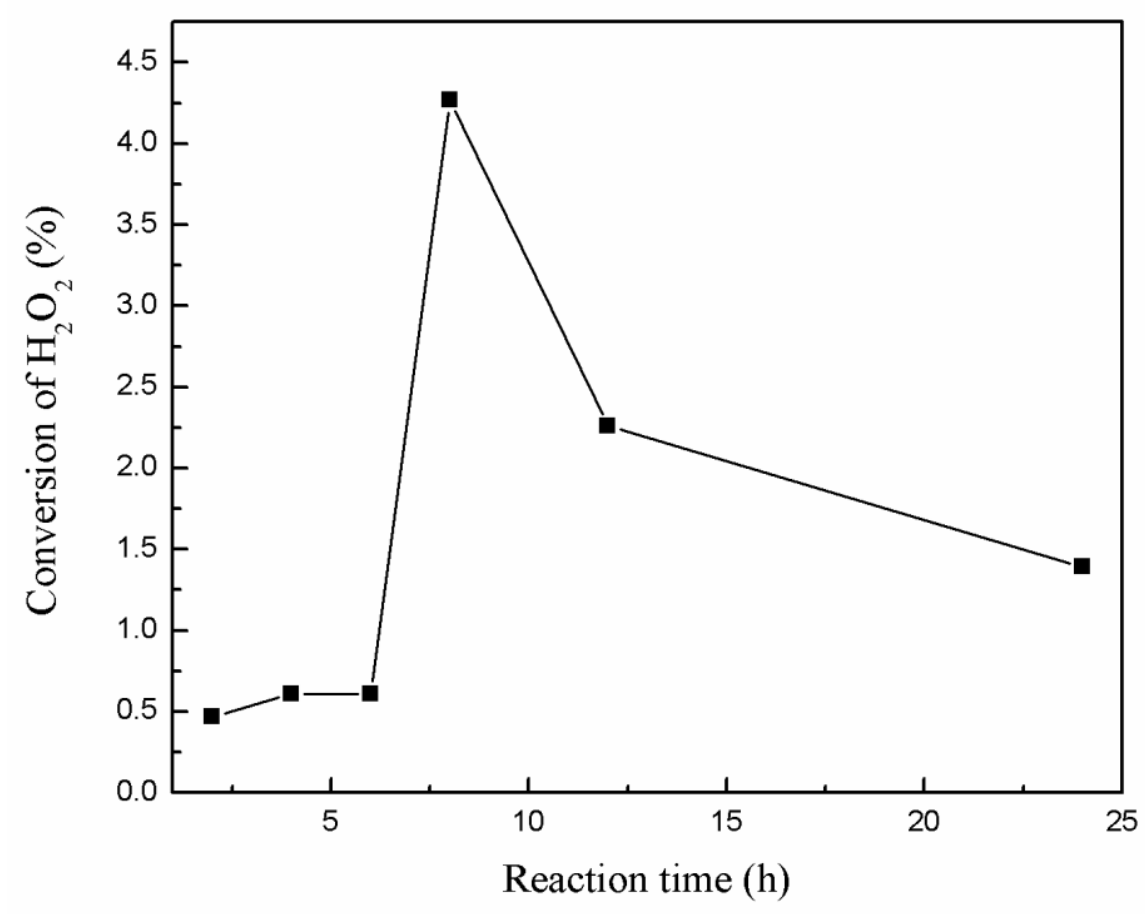

Figure S2. Effect of the reaction time on the conversion of $\mathrm{H}_{2} \mathrm{O}_{2}$. Reaction conditions: benzene (1.0 mL, $11.26 \mathrm{mmol}), 30 \%$ aq $\mathrm{H}_{2} \mathrm{O}_{2}(2 \mathrm{~mL}, 20 \mathrm{mmol})$, Fe/SBA-16 (0.1 g), 
acetonitrile $(6 \mathrm{~mL}, 114.8 \mathrm{mmol}), \mathrm{T}=65^{\circ} \mathrm{C}$.

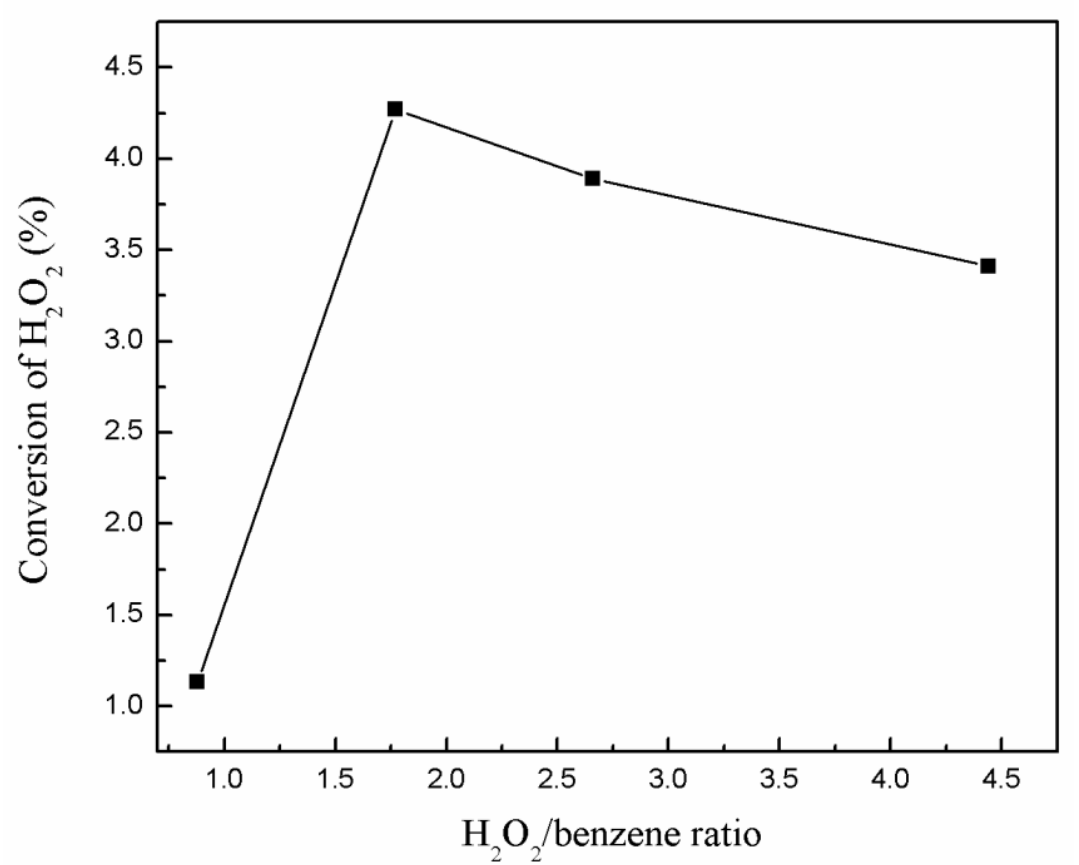

Figure S3. Effect of the amount of $\mathrm{H}_{2} \mathrm{O}_{2}$ on the conversion of $\mathrm{H}_{2} \mathrm{O}_{2}$. Reaction conditions: benzene (1.0 mL, $11.26 \mathrm{mmol})$, Fe/SBA-16 (0.1 g), acetonitrile $(6 \mathrm{~mL}, 114.8 \mathrm{mmol}), \mathrm{T}=65$ ${ }^{\circ} \mathrm{C}, \mathrm{t}=8 \mathrm{~h}$.

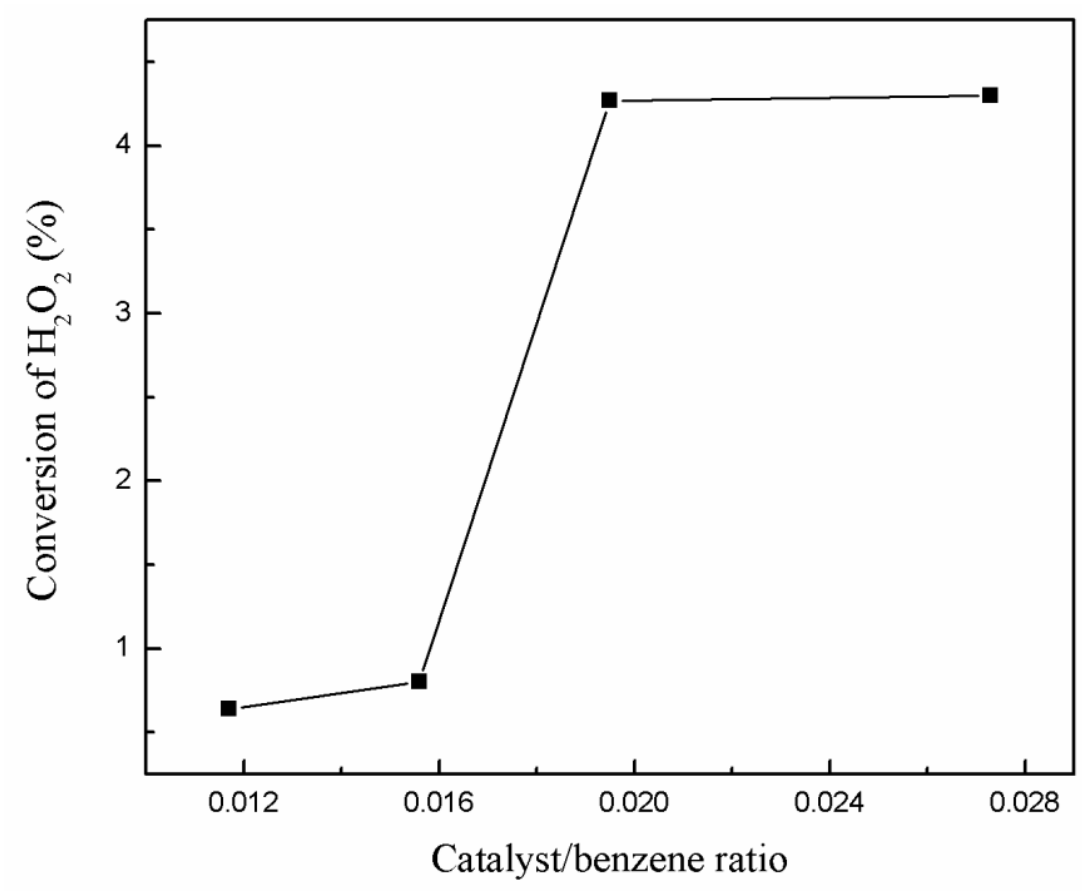

Figure S4. Effect of the catalyst dosage on the conversion of $\mathrm{H}_{2} \mathrm{O}_{2}$. Reaction conditions: benzene (1.0 mL, $11.26 \mathrm{mmol}), 30 \%$ aq $\mathrm{H}_{2} \mathrm{O}_{2}(2 \mathrm{~mL}, 20 \mathrm{mmol})$, acetonitrile $(6 \mathrm{~mL}, 114.8$ $\mathrm{mmol}), \mathrm{T}=65^{\circ} \mathrm{C}, \mathrm{t}=8 \mathrm{~h}$. 